significantly improved diagnosis of infection. However, these molecular tools still have some limitations especially in the case of low parasitaemia. Furthermore, research is still needed to make molecular detection a real control tool for the fight against sleeping sickness. The purpose of this study is to determine the threshold of sensitivity of real-time PCR using the $18 \mathrm{~S}$ and TgsGp primers and of the LAMP technique, applied in the DiTECT-HAT project as molecular reference tests.

Methods We used serial dilutions containing 0, 1, 10, 100, $10^{3}, 10^{4}, 10^{5}, 10^{6}$ parasites per $\mathrm{ml}$ of blood. Samples were extracted, and DNA was amplified.

Results The analytical sensitivity of the $18 \mathrm{~S}$ real-time PCR with the Taqman probe of the filter paper samples is 100 parasites/ml and that of the TgsGp real-time PCR with the Taqman probe of filter paper samples is $10^{4}$ parasites $/ \mathrm{ml}$. For Lamp technique, the analytical sensitivity is $10^{3}$ parasites $/ \mathrm{ml}$.

Conclusion This study shows that a 'negative PCR' would not mean 'no parasite'. It suggests that DNA detection techniques should still be improved.

\section{PO 8444 CHARACTERISATION OF PATHOGENS CAUSING DIARRHOEA IN CHILDREN UNDER FIVE IN LAMBARÉNÉ, GABON}

\begin{abstract}
1,2Gédéon P Manouana*, ${ }^{1,3}$ Gedeon Bingoulou Matsougou, ${ }^{4}$ Natalie Byrne, ${ }^{5}$ Philipp Hofmann, ' $m$ mirabeau Mbong Ngwese, 'Pau A Nguema Moure, 'Jeannot Fréjus Zinsou, 'Jean Claude Dejon Agobe, 'Bayode R Adegbite, 'Jean R Edoa, ${ }^{1}$ Yabo J Honkpehedji, 'Matthew B.B. Mccall, 'Abraham S Alabi, ${ }^{6,7}$ Daniel Eibach, ${ }^{1,2}$ Peter G Kremsner, ${ }^{2}$ Steffen Borrmann, ${ }^{1,2}$ Ayola A Adegnika. ${ }^{1}$ Centre de Recherches Médicales de Lambaréné, Lambaréné, Gabon; ${ }^{2}$ Institut für Tropenmedizin, Universität Tübingen, Tübingen, Germany; ${ }^{3}$ Faculté de médecine, Université des Sciences de la Santé, Libreville, Gabon; ${ }^{4}$ Faculty of Medicine, Universität Tübingen, Germany; ${ }^{5}$ Charité-Universitätmedizin Berlin, Germany; ${ }^{6}$ Bernhard Nocht Institute for Tropical Medicine; ${ }^{7}$ German Center for Infection Research, Hamburg-Borstel-Lübeck, Germany
\end{abstract}

\subsection{6/bmjgh-2019-EDC.99}

Background Diarrhoeal disease remains the second leading cause of death in children under five years, being associated with about 525,000 deaths every year. The most common pathogens worldwide are Shigella spp/EIEC, rotavirus, adenovirus 40/41, ST-ETEC and Cryptosporidium spp. Public health interventions rely on estimates of pathogen-specific burden for prioritisation. Sadly, comprehensive data on the aetiology of diarrhoea in children is lacking for Gabon. This study aimed to identify the spectrum of pathogens found in Lambaréné, Gabon and provide baseline data on their prevalence, needed for implementation of effective control measures.

Methods A cross-sectional study was conducted at Albert Schweitzer and Georges Rawiri Regional hospitals in Lambaréné from February 2017 to February 2018. A consecutive sample of children under 5 year old with diarrhoea or a history of diarrhoea within the previous three days were prospectively studied. A single stool sample was collected from each study participant and processed using commercial rapid immunoassays to detect antigens of rotavirus, adenovirus, and Cryptosporidium spp. Multiplex PCR was used for Cryptosporidium spp., Giardia lamblia and Cyclospora cayetanensis detection, and characterisation of E. coli strains.

Results Out of 188 participants who provided stool samples, one or more pathogens could be detected in $34.6 \%$ of the cases. The most prevalent parasites were Giardia lamblia (14.9\%), Cryptosporidium spp. (11.7\%), and Cyclospora cayetanensis (2.7\%). Enteric viruses also were identified in these children: $10.6 \%$ and $1.6 \%$ of rotavirus and adenovirus, respectively. Multiple pathogens were detected in $5.3 \%$ of samples.

Conclusion This analysis of the causes of diarrhoea in children under 5 years of age in our setting showed three main pathogens: Giardia lamblia, Cryptosporidium spp. and rotavirus. Our study confirms major agents of acute diarrhoeal diseases in children, highlights research needs (Cryptosporidium) and supports the introduction of new tools such as the implementation of the rotavirus vaccine in the national immunisation programme.

\section{PO 8446 COLLABORATIVE TUBERCULOSIS RESEARCH AGENDA AT KEMRI CENTER FOR GLOBAL HEALTH RESEARCH, KISUMU, KENYA}

${ }^{1}$ Steve Wandiga, ${ }^{1}$ Janet Agaya, ${ }^{1}$ Ouma S Gurrion, ${ }^{1}$ Ochieng Albert Okumu, ${ }^{2}$ Grace Kiringa, ${ }^{3} J u l i a n a$ Otieno, ${ }^{4}$ Geoffrey Mwai, ${ }^{2}$ Videlis Nduba, 'Stephen Munga. ${ }^{1}$ Kenya Medical Research Institute - Center for Global Health Research, Kisumu, Kenya; ${ }^{2}$ Kenya Medical Research Institute - Center for Respiratory Diseases Research, Nairobi, Kenya; ${ }^{3} J a r a m o g i$ Oginga Odinga Teaching and Referral Hospital, Kisumu, Kenya; ${ }^{4}$ Siaya County Referral Hospital, Siaya, Kenya

\subsection{6/bmjgh-2019-EDC.100}

Background Developing countries grapple with inadequate funding amidst high burden of diseases. Africa is home to 9 of the 22 countries with a high tuberculosis (TB) burden and to 29 of the 41 countries with a high TB-HIV burden. Kenya is among the high TB and high TB-HIV burden countries. The Western Kenya region has the highest burden of $\mathrm{TB}$ and HIV. North-South partnerships are pivotal in ameliorating funding gaps in clinical research.

Methods While optimising existing infrastructure and organising programme support (i.e sensitisation and awareness creation, leading to study participant recruitment), from 2005 to date we conducted with multiple North-South collaborators capacity strengthening, TB prevalence survey, observational studies, operational research, and vaccine and drug trials.

Results TB prevalence survey showed 600 cases per 100,000 population, TB epidemiological studies among adolescents and infants yielded 680 and 900/100,000 population respectively while $2 \mathrm{~TB}$ vaccine trials among infants and adults were conducted in Siaya. Three TB drug trials and a TB patient observational cum bio-bank study were concluded in Kisumu. KEMRI TB laboratory was upgraded from BSL2 to BSL3, was ISO-accredited in 2013, renewed in 2015 and 2017 and supports TB programme health facilities with retreatment specimens, supervision and mentorship. Over 25 operational TB studies grouped into community and case detection (increasing case detection), diagnostic and molecular (new diagnostic methods) and epidemiology studies (testing and monitoring cohorts for epidemiological questions) were implemented. Five PhDs, 9 Master's, 2 Postgraduate Diplomas, 6 Bachelor's degrees and 10 Diplomas have been supported. Siaya clinical research center was built while in Kisumu an adolescent clinic was constructed. This work involved 18 northern and 26 southern partners. Over 35 publications have been published out of these collaborations.

Conclusion North-South collaborations provided funding, expertise and resources to harness research capacity of KEMRI; hence the need to foster a global networking culture. 